\title{
Carbono acumulado en la biomasa vegetal de la reserva de Villavicencio (Mendoza-Argentina)
}

\author{
LILIANA ZIVKOVIC ${ }^{1,2}$, EDUARDO MARTÍNEZ CARRETERO ${ }^{2}$, A. DALMASSO $^{2}$ y \\ M. ALMIRÓN²
}

\begin{abstract}
Summary: Carbon storage in the plant biomass of the Villavicencio Nature Reserve (MendozaArgentina). Nature reserves play a relevant role in carbon storage in arid lands. The vegetation of the Nature Reserve of Villavicencio stores $98798 \mathrm{Mg}(2.1 \mathrm{Mg} / \mathrm{ha})$ of carbon in total; considering the Puna and unburned Monte vegetation, $91.2 \%$ of carbon occurs in aboveground parts (leaves and stems) and $8.8 \%$ in the root system. Monte vegetation, characterized by Larrea cuneifolia shrubland, stores $77905 \mathrm{Mg} \mathrm{C}(3.9 \mathrm{Mg} / \mathrm{ha}), 78.8 \%$ of the total carbon in the area. Nanophanerophytes store $28.8 \%$ in the unburned shrubland, and $50.4 \%$ in the area burned in 2000 , while chamaephytes store $95.5 \%$ in the area burned in 2005. In the Puna belt, with $20893 \mathrm{Mg} \mathrm{C}(0.78 \mathrm{Mg} / \mathrm{ha})$ stored, Jarava vaginata grassland dominates and stores $88 \%$ of carbon, aboveground parts (leaves of grasses and leaves and stems of chamaephytes) store $90 \%$ of carbon. In general, and taking into account both vegetation belts, $19.8 \%$ of the carbon present in the Reserve is stored in nanophanerophytes, $32.8 \%$ in hemicryptophytes, $28.8 \%$ in chamaephytes and $18.4 \%$ in succulents (cactaceae). Assessment of carbon storage, taking into account the different phytogeographic units, is necessary for management of the protected area and for a better understanding of the role of these environments in the mitigation of atmospheric carbon.
\end{abstract}

Key words: Carbon, life forms, protected area.

Resumen: Las reservas naturales en las zonas áridas cumplen un papel destacado en el almacenamiento de carbono. La vegetación de la Reserva de Villavicencio acumula en total $98798 \mathrm{Mg}(2,1 \mathrm{Mg} / \mathrm{ha}) \mathrm{de}$ carbono; considerando la vegetación de la Puna y del Monte no quemado el $91,2 \%$ del carbono se encuentra en la parte aérea (hojas y tallo) y el 8,8 \% restante en el sistema radical. La vegetación del Monte, caracterizada por el matorral de Larrea cuneifolia, acumula $77905 \mathrm{Mg}(3,9 \mathrm{Mg} / \mathrm{ha}), 78,8 \%$ del carbono total del área. En el matorral no quemado las nanofanerófitas acumulan el $28,8 \%$, en el área incendiada en el 2000 el 50,4\% y en la quemada en el 2005 las caméfitas el 95,5\%. En la vegetación del piso de la Puna, con $20893 \mathrm{Mg}$ de carbono (0,78 Mg/ha) Almacenado, domina el pastizal de Jarava vaginata que acumula el $88 \%$ del carbono; la parte aérea (follaje de gramíneas y hojas y tallos de caméfitas) almacena el $90 \%$ del carbono. En general, y considerando ambos pisos de vegetación, 19,8\% del carbono presente en toda la Reserva lo acumulan las nanofanerófitas, las hemicriptófitas el 32,8\%, las caméfitas el $28,8 \%$ y las suculentas (cactáceas) el 18,4\%. La evaluación del carbono acumulado, considerando las diversas unidades de vegetación, es necesaria para el manejo del área protegida y para una mejor comprensión del papel de estos ambientes en la mitigación del carbono atmosférico.

Palabras clave: Carbono, formas de vida, área protegida.

\section{INTRODUCCIÓN}

El manejo del carbono, a través del manejo de la biomasa vegetal, acumulado en diferentes paisajes

1 Università Politecnica delle Marche, Ancona, Italia. lilizivko@hotmail.com

2 Geobotánica y Fitogeografía, IADIZA (CONICET), CC 507, 5500 Mendoza. mcarrete@mendoza-conicet.gob.ar es un aspecto relevante en la mitigación de la contaminación atmosférica y el cambio climático. Según Campbell et al. (2008), el conocimiento del carbono acumulado en áreas protegidas juega un papel destacado en las estrategias para reducir las emanaciones de $\mathrm{CO} 2$ causadas por la deforestación, en este sentido las áreas protegidas con muy bajos impactos antrópicos cumplen una importante función a nivel local y regional, más aún en regiones áridas 
(Lal, 2002). Las reservas naturales al mantener su estructura y función poco o nada afectadas por acción humana resultan en ambientes adecuados para obtener datos de referencia ante los procesos de desertificación y el cambo climático (Reynolds et al, 2005; Verón et al., 2006). En Argentina es aún escaso el conocimiento del carbono acumulado en las áreas protegidas de las zonas semiáridas y áridas (Gasparri \& Manghi, 2004; Gasparri et al., 2007). Iglesias et al. (2012) evaluaron el carbono acumulado en tres áreas protegidas del centro-oeste de Argentina siguiendo un gradiente de precipitaciones.

El carbón almacenado y el flujo entre parte aéreasubterránea ha sido estudiado en sistemas boscosos tropicales principalmente para entender su papel en el ciclo global del carbono (Malhi \& Grace, 2000) o para su potencial empleo en bonos de carbono (Glenday, 2006). Por otra parte, la magnitud del carbono almacenado en la parte subterránea de los pastizales es un componente relevante en el ciclo global (Schuman et al., 2002).

El cambio de uso de la tierra, principalmente deforestación, contribuye con aproximadamente el $20 \%$ de la emisión de $\mathrm{CO}_{2}$ (IPCC, 2007). El mayor potencial en la mitigación en la acumulación de carbono proviene, por una parte, de eliminar o limitar la creciente tasa de deforestación (Watson et al., 2000), y por la otra en el manejo de las zonas no cultivables y en la conservación de las áreas protegidas. Gasparry \& Manghi (2004) estiman que la deforestación es la principal causa de emisión de carbono a la atmósfera en Argentina, donde sólo los bosques extratropicales del norte argentino emiten anualmente $20875 \mathrm{Mg}$ de carbono (Gasparry et al., 2008).

El carbono almacenado ha sido estudiado principalmente en ecosistemas con elevado contenido en biomasa (bosques templados), pero poco se ha avanzado en evaluar el carbono acumulado en ambientes áridos o semiáridos (Bonino, 2006), a pesar de que estos ambientes cubren aproximadamente el $30 \%$ del área continental global (Bechtold \& Inouye, 2007). Para el matorral central semiárido de Chile Quezada et al. (2011) evaluaron una acumulación de $36,5 \mathrm{Mg} \mathrm{C} / \mathrm{ha}$.

El secuestro de carbono es un aspecto relevante de las áreas protegidas, especialmente las que incluyen bosques y pastizales por cuanto disponen de mayor cobertura y biomasa vegetal (Coupland, 1992). De esta manera estas áreas constituyen un componente importante en las estrategias de conservación y de mitigación del efecto del cambio climático por incremento del carbono atmosférico y para ello deben reconocerse las necesidades del entorno social en cuanto, por ejemplo, las compensaciones por servicios ambientales (Brandon \& Wells, 1992), así como los beneficios económicos de la conservación y mitigación por las reservas y parques (Kulshreshtha \& Johnston, 1991; Hadker et al., 1997; Driml, 1997). Campbell et al. (2008) estimaron que globalmente los ecosistemas incluidos en las áreas protegidas almacenan más de 312 Gt de carbón, aproximadamente el 15,2\% del carbón terrestre acumulado. Otra forma de avaluar el carbono acumulado en las áreas protegidas es considerando las categorías fijadas por la IUCN, según UNEP-WCMC (2008) se acumulan 87Gt en las de categoría I-II, 139Gt en las de categoría I-IV y 233Gt en las de categoría I-VI.

Por otra parte, resulta relevante identificar los tipos funcionales de las plantas y su respuesta a diversas condiciones ambientales (Smith et al., 1997; Woomer et al., 2004). Diversos estudios muestran asociación positiva entre carbono almacenado, cobertura del suelo y tipos funcionales de plantas (Caylor et al., 2003; Ni, 2003).

La Reserva Natural Villavicencio, comprendida en la Categoría IV de IUCN, se ubica en el noroeste de Mendoza y ocupa dos grandes unidades morfoestructurales: la Precordillera y el Piedemonte precordillerano.

Desde el punto de vista fitogeográfico tres provincias se encuentran en el área de Villavicencio: la del Monte entre los 1500 y 1600 m (Roig et al., 2009), del Cardonal principalmente en laderas de exposición norte (Dalmasso et al., 1999) y la Puna entre los 2700 y 3000 m que alcanza a esta latitud su límite austral de distribución (Roig \& Martínez Carretero, 1998; Martínez Carretero, 1995, 2001). La vegetación del Monte se presenta como un matorral bajo y abierto de Larrea divaricata o L. cuneifolia de hasta $1 \mathrm{~m}$ de alto, la de la Puna como un pastizal muy bajo dominado por Jarava vaginata Phil. En el Monte, el piso inferior de L. cuneifolia, suele sufrir incendios que en ocasiones alcanzan importantes extensiones y que determinan un cambio en la estructura y composición florística, dominando $S$. teuissima Trin. o $S$. eriostachya Kunth según la altitud (Martínez Carretero, 1984, 1987). En el piso de Puna dominan las hemicriptófitas mientras que en el Monte las nanofanerófitas. Luego de los incendios periódicos, en el Monte la fisonomía cambia de matorral abierto a pastizal con dominio de las 


\section{Zivkovic et al. - Carbono acumulado en la biomasa vegetal de Mendoza}

hemicriptófitas (Martínez Carretero, 1984).

La acumulación de carbono en la vegetación del Monte ha sido estudiada a nivel regional por Martínez Carretero \& Dalmasso (2007), quienes determinaron que en el piedemonte de la Precordillera las dos especies dominantes, L. cuneifolia y $L$. divaricata, acumulan cada una 12000 y $130000 \mathrm{Mg}$ de carbono respectivamente.

En este trabajo se busca determinar el carbono acumulado por la vegetación de las dos unidades fitogeográficas más extensas en la Reserva Natural Villavicencio, el Monte y la Puna, y según el estado de conservación de la vegetación: quemado y no quemado, tanto en la parte aérea como radical, teniendo en cuenta las formas biológicas

\section{Materiales y Métodos}

Área de estudio

La Reserva de Villavicencio abarca 62.000 ha (32 $43^{\prime} 37^{\prime \prime} \mathrm{S}-69^{\circ} 09^{\prime} 38^{\prime \prime} \mathrm{W} / 32^{\circ} 25^{\prime} 12^{\prime \prime} \mathrm{S}-$ $\left.68^{\circ} 48^{\prime} 33^{\prime \prime} \mathrm{W}\right)$, altimétricamente se ubica entre los 1500 y $3200 \mathrm{~m}$. Los registros climáticos para las localidades de Paramillos (3200 m), Sitio de Antena (2000 m) y Restaurante $(1800 \mathrm{~m})$ indican 69,220 y $571 \mathrm{~mm}$ de lluvia respectivamente; el mayor porcentaje ocurre en el período de verano (Capitanelli, 1967). Según De Fina (1992) para Villavicencio a $1780 \mathrm{~m}$ la temperatura media del mes de Enero (más cálido) es de $17^{\circ} \mathrm{C}$ y la Julio (más frío) $5^{\circ} \mathrm{C}$. En la localidad de Las Higueras $(1125 \mathrm{~m})$, en el límite noreste de la reserva, la temperatura media del mes de enero es de $21,3^{\circ} \mathrm{C}$ y la de julio $6,7^{\circ} \mathrm{C}$. En la Fig. 1 se indica el área protegida y las unidades fitogeográficas evaluadas.

\section{Toma de datos}

En cada unidad fitogeográfica se establecieron al azar: 10 parcelas de $5 \times 20 \mathrm{~m}$ en el Monte y 5 de $10 \times 20 \mathrm{~m}$ en la Puna. En el Monte, cuatro parcelas se ubicaron en el área no incendiada, tres en el área incendiada en el año 2000 y tres en la incendiada en el 2005. En cada parcela se registró la cobertura específica y la cobertura de mantillo mediante el método de puntos de intersección modificado (Passera et al., 1983), considerando dos transectas en diagonal de $20 \mathrm{~m}$ cada una, las lecturas se hicieron cada $10 \mathrm{~cm}$. En cada parcela se determinó por conteo la densidad de cada especie y se colectaron todos los ejemplares de cada especie con su sistema radical lo más completo posible. Se estableció la forma biológica por especie. En cada planta se separó raíz, tallos y hojas; todo el material se llevó a estufa de circulación forzada a $60^{\circ} \mathrm{C}$ durante 48-72 horas (hasta peso constante) y luego se pesó en balanza digital Mettler sensibilidad 0,01 g. Para transformar los datos de peso seco a carbono se aplicó el coeficiente 0,5 (Kobak, 1988; Isaev et al., 1993), que corresponde al porcentaje de carbono en la materia seca. La superficie ocupada por cada unidad fitrogeográfica, como la de las áreas incendiadas, se obtuvo a través del análisis de imagen satelital LANDSAT 7 TM empleando el software ArcGis 9.3. La biomasa seca media (media de todas las parcelas por unidad fitogeográfica) de raíz, tallo y hojas obtenida por $\mathrm{m}^{2}$ se multiplicó por la superficie total de cada unidad fitogeográfica y se obtuvo la biomasa particionada y el carbono acumulado, por especie y por forma biológica.

\section{Resultados}

De las 46051 ha analizadas en la Reserva, la Puna ocupa 26475 ha (57,5\%) y el Monte 19576 ha (42,5 $\%)$. El área incendiada en el año 2000 alcanzó a 1834 ha (9,4 \% del total del Monte), mientras que el incendio del 2005 alcanzó a 265 ha (1,4\%).

\section{Cobertura especifica}

La vegetación del Monte se presenta como un matorral abierto con cobertura media del 31,38\%, $57,5 \%$ de suelo desnudo y $11,12 \%$ de cobertura de mantillo. La especie dominante por cobertura es Larrea cuneifolia (8,8\%), acompañada por Zuccagnia punctata Cav. (2\%) y Pappophorum caespitosum (2\%). L. cuneifolia alcanza a 4400 individuos por hectárea, Tephrocactus articulatus a 900, Echinopsis leucantha (Gillies ex Salm-Dyck) Walp. a 300, Cereus aethiops Haw. a 100, Trichocereus strigossus (SalmDyck) Britton \& Rose a 100, entre otras (Tabla 1). La nanofanerófita es la forma biológica dominante.

\section{Sector incendiado el año 2000}

Luego de 9 años de ocurrido el último incendio la fisonomía corresponde a la de un pastizal con cobertura vegetal media del 48,7\%, 29\% de suelo desnudo y $22,23 \%$ de cobertura de mantillo. Dominan Acantholippia seriphioides (A. Gray) Moldenke 


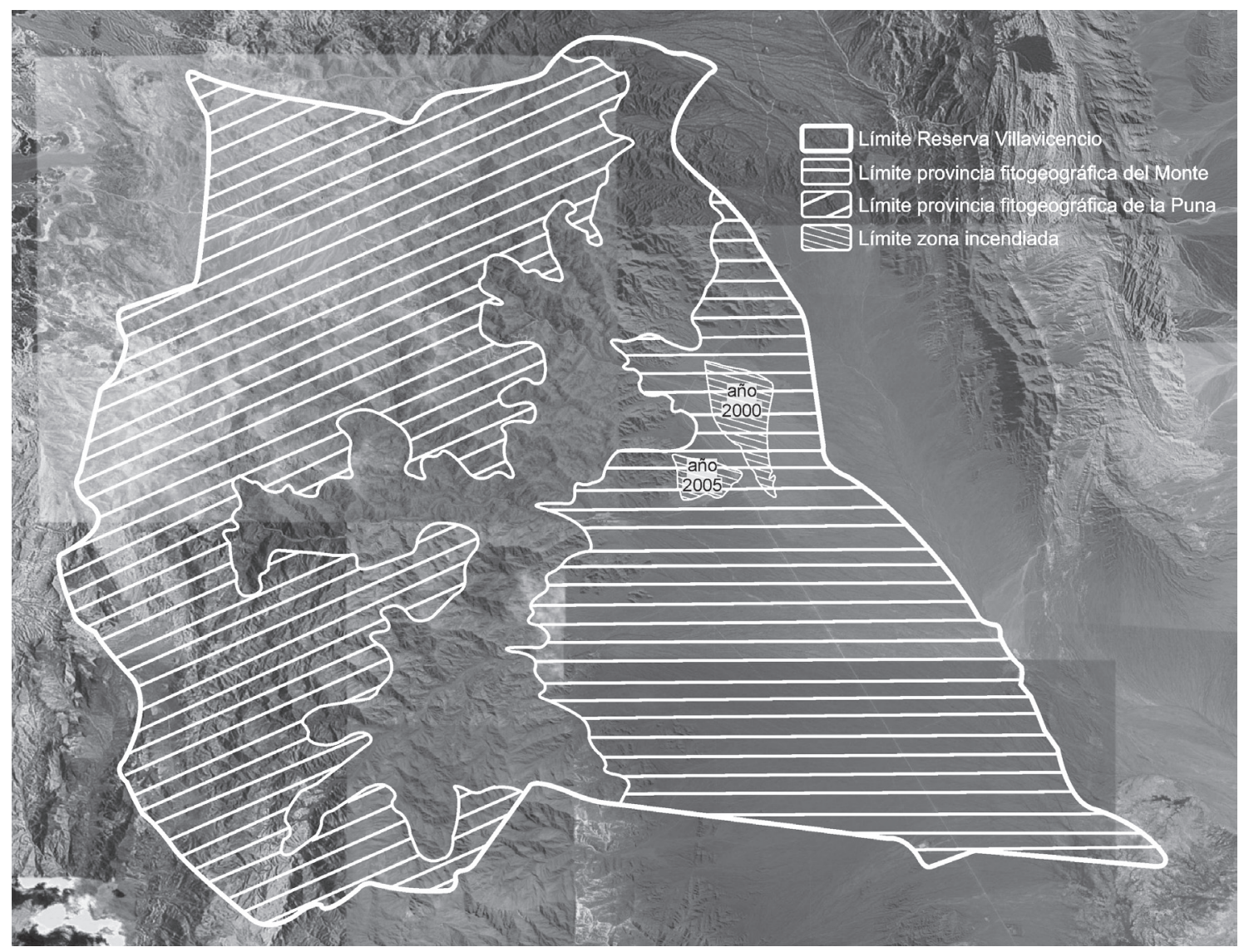

Fig. 1. Reserva de Villavicencio y distribución del Monte y Puna.

(28\% cobertura, 31 individuos/ha), Jarava ichu Ruiz \& Pav. (6,7\% de cobertura y 5300 individuos/ha) y Pappaphorum caespitosum (6,4\% cob., 100 indiv./ha), acompañada por Diplachne dubia (H.B.K.) Scribner (3,6\% cob., 2200 indiv./ha.) Las hemicriptófitas presentan la mayor cobertura acompañadas por nanofanerófitas y caméfitas.

\section{Sector incendiado el año 2005}

Este sector no presentó incendios periódicos por lo que después de cuatro años de quemado la fisonomía es la de un pastizal con arbustos bajos esparcidos. La cobertura vegetal media es del $45,5 \%$, el suelo desnudo alcanza al $33,67 \%$ y el mantillo al $20,83 \%$. La especie dominante es $J$. ichu (20,33\% de cobertura, 9100 indiv./ha) acompañada por Larrea cuneifolia (9,83\% cob., 1300 indiv./ha) en su mayoría con plantas quemadas.

La Puna se presenta fisonómicamente como un pastizal muy bajo y abierto con escasos arbustos bajos. La cobertura vegetal media es del $35 \%$, el mantillo cubre el $12 \%$ y el suelo desnudo alcanza al 53\%. J. vaginata alcanza al 20\% de cobertura y 1246 indiv./ha, acompañada por Maihueniopsis glomerata (Haw) Kiesling con el $8 \%$ cob. y 25 indiv./ha y $A$. mendozana var. paramilloensis y Ephedra chilensis Miers con el 6\% cob. cada una y 355 y 110 indiv./ha respectivamente. Las hemicriptófitas dominan y hacen a la fisonomía.

\section{Formas biológicas}

En el Monte no quemado dominan las nanofanerófitas con el 55\% de cobertura, codominando las hemicriptófitas con el $11 \%$ y las suculentas (cactáceas) con el 6\%.

En el área incendiada en el año 2000, con incendios recurrentes hasta esa fecha, dominan las hemicriptófitas con el $70 \%$ de cobertura acompañadas 
Tabla 1. Cobertura específica (\%) y número de individuos por hectárea, de las especies más conspicuas, en las comunidades estudiadas.

\begin{tabular}{|c|c|c|c|c|c|c|c|c|}
\hline \multirow[t]{3}{*}{ Especie } & \multicolumn{6}{|c|}{ Monte } & \multirow[b]{3}{*}{$\begin{array}{l}\text { Cob. } \\
-\%-\end{array}$} & \multirow{3}{*}{$\begin{array}{l}\text { Puna } \\
\mathrm{N}^{\circ} \text { indiv./ha }\end{array}$} \\
\hline & \multicolumn{2}{|c|}{ Matorral no quemado } & \multicolumn{2}{|c|}{ Incendio 2000} & \multicolumn{2}{|c|}{ Incencio 2005} & & \\
\hline & $\begin{array}{c}\text { Cob. } \\
-\%-\end{array}$ & $\mathrm{N}^{0}$ indiv./ha & $\begin{array}{l}\text { Cob. } \\
-\%-\end{array}$ & $N^{0}$ indiv./ha & $\begin{array}{l}\text { Cob. } \\
-\%-\end{array}$ & $\mathrm{N}^{0}$ indiv./ha & & \\
\hline Larrea cuneifolia & 8 & 4400 & 0,5 & 13 & 9,8 & 1300 & - & - \\
\hline Tephrocactus articulatus & 1 & 900 & - & - & - & - & - & - \\
\hline Pappophorum caespitosum & 5 & 3200 & 6,4 & 100 & 5 & 4600 & - & - \\
\hline Junellia aspera & 1,3 & 800 & - & - & 3,6 & 800 & - & - \\
\hline Acantholippia seriphioides & 1 & 300 & 27,8 & 31 & 6 & 3100 & - & - \\
\hline Jarava ichu & 1 & 1100 & 6,6 & 5300 & 20,3 & 9100 & - & - \\
\hline Echinopsis leucantha & 1 & 300 & - & - & - & - & - & - \\
\hline Cereus aethiops & 1 & 100 & - & - & - & - & - & - \\
\hline Trichocereus strigossus & 1 & 100 & - & - & - & - & - & - \\
\hline Lycium chilense & 1 & 100 & - & - & 3,6 & 500 & - & - \\
\hline Diplachne dubia & - & - & 3,6 & 2200 & - & - & - & - \\
\hline Menodora decemfida & - & - & 4 & 1400 & - & - & - & - \\
\hline Nasella tenuissima & - & - & - & - & 4,5 & 600 & - & - \\
\hline Jarava vaginata & - & - & - & - & - & - & 20 & 1246 \\
\hline Maihueniposis glomerata & - & - & - & - & - & - & 8 & 25 \\
\hline Ephedra chilensis & - & - & - & - & - & - & 6 & 110 \\
\hline Artemisia mendozana var. & - & - & - & - & - & - & 6 & 355 \\
\hline
\end{tabular}

por las nanofanerófitas con el $18 \%$ y las caméfitas con el $11 \%$.

En el área incendiada en el año 2005 las hemicriptófitas alcanzan al $64 \%$ de cobertura, las caméfitas el 38\% y las terófitas el 14\%, aunque por presencia son similares con el 50,4 y $48,4 \%$ de los individuos, respectivamente.

En la Puna dominan las hemicriptófitas con el $20 \%$ de cobertura, seguidas por las caméfitas con un $14 \%$; sin embargo, por presencia dominan las hemicriptófitas.

\section{Biomasa}

La biomasa seca media aérea y subterránea, en kilogramos por hectárea, y por forma biológica para Monte y Puna se indica en la Tabla 2.

En la Tabla 3 se indica el carbono acumulado considerando la biomasa obtenida de la parte radical, follaje y tallo, y según la superficie ocupada por cada unidad fitogeográfica en la Reserva.

En el Monte, en el matorral no quemado, las nanofanerófitas acumulan el $28.8 \%(1238 \mathrm{Mg})$ y las cactáceas el $68.4 \%(2936 \mathrm{Mg})$ del carbono, en el área incendiada en el año 2005 el 95,5\% (10313 $\mathrm{Mg}$ ) las caméfitas y en la del incendio del año
2000, área sometida a quemas periódicas, el 50,4\% $(3,2 \mathrm{Mg})$ las nanofanerófitas y el 35,4\% (2,25 Mg) las hemicriptófitas. En la Puna las hemicriptófitas acumulan el 88,9\% (714 Mg) del carbono y en ellas el 94\% en la parte aérea.

Para toda la reserva el carbono acumulado es de $98798 \mathrm{Mg}(2,1 \mathrm{Mg} / \mathrm{ha})$ de los cuales la vegetación de la Puna representa el 21,2\% (20893 Mg - $0,78 \mathrm{Mg} / \mathrm{ha}-)$ y la del Monte el 78,8\% (77905 Mg -3,9 Mg/ha-). Del total del carbono acumulado, en promedio, el $69,1 \%$ ocurre en la parte aérea de las plantas y el 30,9\% en la subterránea. Considerando cada unidad fitogeográfica por separado, en la Puna el carbono de la parte aérea representa el 90,5\% mientras que la subterránea el $9,5 \%$. En el Monte, en el matorral no incendiado en la parte aérea se acumula el $91,8 \%$ del carbono y el $8,2 \%$ en la subterránea; en el área incendiada en el año 2000 el $82,7 \%$ en la parte aérea y el $17,3 \%$ en la subterránea y en la incendiada en el 2005 el $11,5 \%$ y $88,5 \%$ en la aérea y subterránea respectivamente.

A nivel específico, en el matorral no quemado L. cuneifolia acumula el 58,8\% del carbono, en el matorral pos fuego del año 2000 Lycium tenuispinosum Miers el 36\%, Jarava ichu el 21\% y Acantholippia seriphioides (A. Gray) Moldenke el 12\% y en el 
Tabla 2. Peso medio (kgMS/ha) de parte aérea y subterránea según forma biológica.

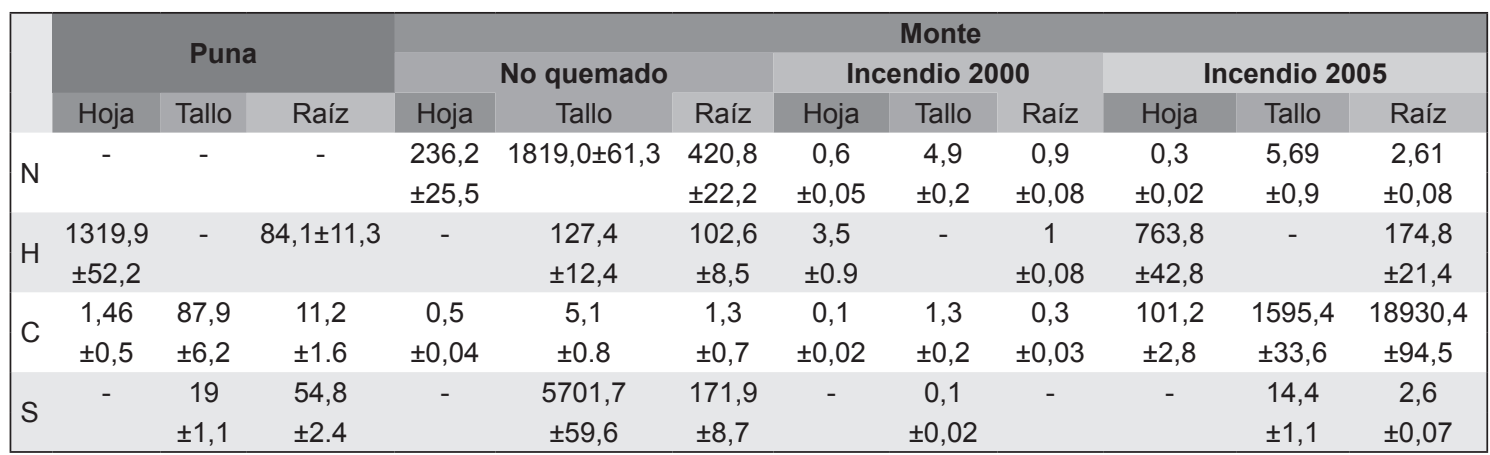

Tabla 3. Carbono acumulado (Mg) en la Puna y el Monte de la Reserva Villavicencio.

\begin{tabular}{|lcccccc|}
\hline & Puna & Matorral no quemado & Incendio 2000 & Incendio 2005 & Total & Total Reserva \\
Parte aérea & 18906 & 68948 & 9,64 & 328 & 77905 & 88192 \\
Parte subterránea & 1987 & 6085 & 2,36 & 2532 & 10606 \\
Total & 20893 & 75033 & 12 & 2860 & 98798 \\
\hline
\end{tabular}

matorral posfuego del año 2005 L. divaricata el $64 \%$ y $S$. ichu el $20,3 \%$. En la Puna el $89,5 \%$ lo acumula $J$. vaginata.

\section{Discusión y Conclusiones}

Considerando la superficie ocupada por matorrales a nivel global Argentina es el país con mayor extensión con esta formación vegetal (Sanderson et al., 2002), que además duplicaría la cantidad de carbono almacenado con respecto a los sistemas forestales y es la principal fuente extratropical de carbón a la atmósfera por deforestación (Gasparri et al., 2008).

En el piedemonte de la Reserva Villavicencio los disturbios antropogénicos como la deforestación mediante quemas periódicas del estrato arbustivo llevan a un pastizal denso y a mayor variabilidad en el paisaje (Martínez Carretero, 1984). Este impacto es relevante pues las especies dominantes $L$. divaricata y L. cuneifolia requieren de un recambio de entre 18 y 16,7 años, respectivamente (Martínez Carretero \& Dalmasso, 2002), por lo que lograr acumular el mismo carbono requiere de ese lapso de tiempo. Iglesias et al. (23012) encontraron similar variabilidad espacial y cambio en el dominio de los tipos funcionales en las reservas de Quebracho de
La Legua y Chancani, asociadas principalmente a fuegos y tala. En Villavicencio, en el piso del Monte, el cambio de fisonomía lo evidencia el dominio de los nanofanerófitos en el matorral sin quemar a los hemicriptófitos en el pastizal derivado del matorral con incendios repetidos. En estos matorrales la mayor parte del carbono (entre el 80 y 90\%, según el estado de conservación) se acumula en la parte aérea (tallos y hojas). En la Puna, en cambio, el impacto está dado por la ganadería caprina extensiva, que en la zona de la Reserva no ocurre o es mínima y ocasional. En ésta región, fría y con vientos constantes, dominan las hemicriptófitas de muy baja altura y con escaso desarrollo radical por el congelamiento estacional del suelo por lo que prácticamente el $90 \%$ del carbono se encuentra en el follaje de las gramíneas. En la Fig. 2 se muestra la partición del carbono entre parte aérea y subterránea según forma biológica y piso de vegetación.

Los contenidos de carbono acumulado son relativamente bajos en comparación con otras áreas protegidas de zonas áridas y semiáridas de Argentina como el bosque maduro de Prosopis flexuosa en la Reserva Natural Telteca con 48 $\mathrm{Mg} / \mathrm{ha}$ y $13 \mathrm{Mg} / \mathrm{ha}$ en el matorral, o el bosque maduro de Aspidosperma quebracho-blanco con $65 \mathrm{Mg} / \mathrm{ha}$ y el matorral con $10 \mathrm{Mg} / \mathrm{ha}$ en la Reserva Chancani (Iglesias et al., 2012). A pesar de ello, los 

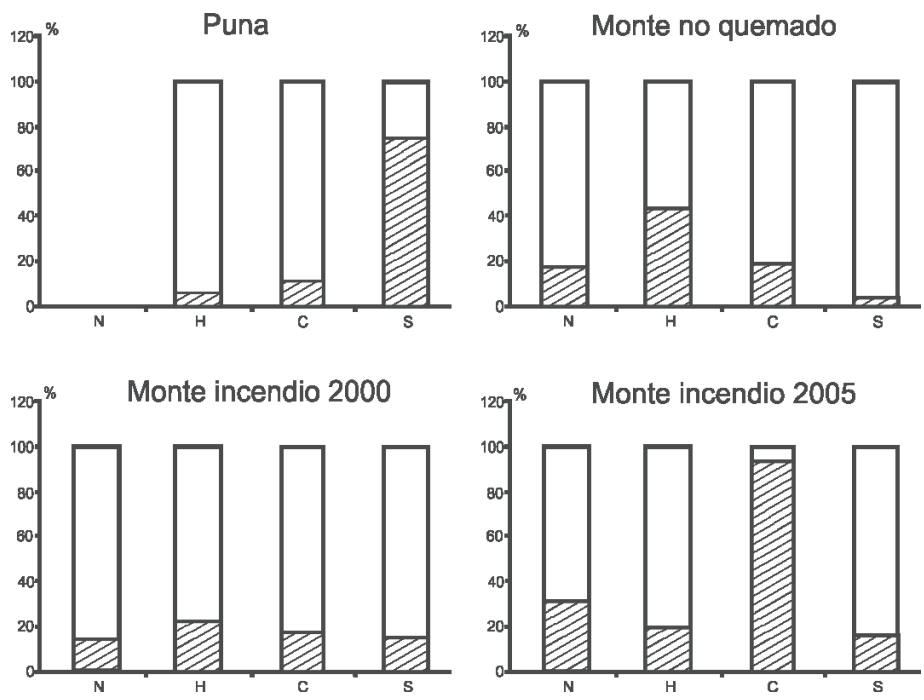

Fig. 2. Partición de carbono en parte aérea y subterránea , según forma biológica y unidad de vegetación. N nanofanerófita, C caméfita, $\mathrm{H}$ hemicritófita, $\mathrm{S}$ suculenta (cactácea).

resultados evidencian la importancia de las áreas protegidas en zonas áridas en términos de secuestro de carbono, por lo que el sistema de manejo de las reservas debería considerar estas funciones ecosistémicas entre los objetivos prioritarios. El principal mecanismo de conservación del carbono acumulado en la vegetación, particularmente de zonas áridas, es prevenir el deterioro de la biomasa de las especies leñosas perennes (nanofanerófitas) evitando el sobrepastoreo, la tala o las quemas periódicas indiscriminadas.

\section{Agradecimientos}

A la administración y a los Guardaparques R. Tobares, C. Robledo y P. Berlanga de la Reserva Natural Villavicencio por facilitar y colaborar en las tareas de campo, a N. Horak y a los revisores por sus sugerencias.

\section{Bibliografía}

BECHTOLD, H.A. \& R.S INOUYE. 2007. Distribution of carbon and nitrogen in sagebrush steppe after six years of nitrogen addition and shrub removal. J. Arid Environ. 71: 122-32.
BONINO, E.E. 2006. Changes in carbon pools associated with a land-use gradient in the Dry Chaco, Argentina. Forest Ecol. Manag. 223: 183-89.

BRANDON, K.E. \& M. WELLS. 1992. Planning for people and parks: Design dilemmas. World Develop. 20: $557-570$.

CAMPBELL, A., L. MYLES, L. LYSENKO, I. HUGHES \& A. GIBBS. 2008. Carbon storage in protected areas-Technical report. UNEP- World Conservation Monitoring Centre.

CAPITANELLI, R. 1967. Climatología de Mendoza. Bol. Est. Geogr. 14: 1-441.

CAYLOR, K.K., H.H. SHUGART, P.R, DOWTY \& T.M. SMITH. 2003. Tree spacing along the Kalahari transect in southern Africa. J. Arid Environ. 54: 281-296.

COUPLAND, R. T. 1992. Natural grasslands, Introduction and Western hemisphere. Ecosystems of the World 8-A. Elsevier, Amsterdam, New York.

DAGET, P. H. \& J. POISSONET. 1971. Une méthode d'analyse phytologique des praires. Critres d'application. Ann. Agron. 22: 5-41.

DALMASSO, A., E. MARTINEZ CARRETERO, F. VIDELA, S. PUIG \& R. CANDIA. 1999. Reserva Natural Villavicencio (Mendoza, Argentina). Plan de Manejo. Multequina 8: 11-50.

DE FINA, A. L. 1992. Aptitud Agroclimática de la Argentina. Academia Nacional de Agronomía y Veterinaria, Buenos Aires.

DRIML, S. 1997. Bringing Ecological Economics out of the Wilderness. Ecol. Econ. 23: 145-153. 
GASPARRI, N.I. \& E. MANGHI. 2004. Estimación de volumen, biomasa y contenido de carbono en las regiones forestales argentinas. http://www.ambiente. gov.ar/archivos/web/UMSEF/File/volumen biomasa_carbono.pdf

GLENDAY, J. 2006. Carbon storage and emissions offset potential in an East African tropical rainforest. Forest Ecol. Manag. 235: 72-83.

GASPARRI, N.I., M.G. PARMUCHI, J. BONO, H. KARZSENBAUM \& C.L. MONTENEGRO. 2007. Utilidad de imágenes LANDSAT 7 TM+ de diferentes fechas para la estimación de biomasa aérea en bosques subtropicales secos de Argentina. XII Congreso de la Asociación Española de Teledetección.

GASPARRI, N.I., H. R. GRAU \& E. MANGHI. 2008. Carbon pools and emissions from deforestation in extra-tropicalforests of northern Argentina between 1900 and 2005. Ecosystems 11: 1247-1261.

HADKER, N., SHARMA, S., DAVID, A. \& T.R. MURALE EDHARAN. 1997. Willingness-to-pay for Borivli National Protected area: Evidence from a Contingent Valuation. Ecol. Econ. 21: 5-122.

IGLESIAS, M. del R., A. BARCHUK \& M. GRILLI. 2012. Carbon storage, community and canopy cover: A comparison along a precipitation gradient. Forest Ecol. Manag. 265: 218-229.

IPCC. 2007. Climate Change 2007: The Physical Sciences Basis. Contribution of Working Group I to the Fourth Assessment Report of the Intergovernmental Panel on Climate Change. In: SALOMON, S., D. QUIN, M. MANNING, Z. CHEN, M. MARQUIS, K.B. TIGNOR \& H.L. MILLER (eds.). Cambridge University Press, Cambridge and New York.

ISAEV, A. S., G. N. KOROVIN, A.I. UTKIN, A. PRYASHNIKOV \& D.G. ZAMOLODCHIKOV. 1993. The estimation of storage and annual carbon deposition in phytomass of forest ecosystems of Russia. Lesovedenie 5: 3-10 (en Ruso).

KOBAK, K. 1988. Biotic components of carbon cycles. Hydrometeoizdat, Leningrad (en Ruso).

KULSHRESHTHA, S. \& M. JOHNSTON. 1991. Economic value of stored carbon in protected areas: A case study of Canadian national parks. www.sampaa.org/publications/conferenceproceedings-1991-2000.

LAL, R. 2002. Carbon sequestration in dryland ecosystems of West Asia and North Africa. Land Degrad. Dev. 14: 45-59.

MALHI, Y. \& J. GRACE. 2000. Tropical forests and atmospheric carbon dioxide. Trends Ecol. Evol. 15: 332-337.

MARTÍNEZ CARRETERO, E. 1984. El incendio de la vegetación en la precordillera mendocina III. Los pastizales disclimáxicos de la quebrada de
Villavicencio. Parodiana 3: 175-183.

MARTÍNEZ CARRETERO, E. 1987. El incendio de la vegetación en la Precordillera mendocina V. Perdida de la calidad nutritiva del sistema natural. Parodiana 5:121-134

MARTÍNEZ CARRETERO, E. 1995. La Puna argentina: Delimitación general y división en distritos florísticos. Incluye mapa 1:2500000. Bol. Soc. Argent. Bot. 31: 27-40.

MARTÍNEZ CARRETERO, E. 2001. Vegetación de los Andes Centrales de la Argentina. El Valle de Uspallata, Mendoza. Bol. Soc. Argent. Bot. 34: $127-$ 148.

MARTÍNEZ CARRETERO, E. \& A. DALMASSO. 2002. Response to cutting of Larrea divaricata and L. cuneifolia in Argentina. Appl. Veget. Sci. 5: 127133.

MARTÍNEZ CARRETERO, E. \& A. DALMASSO. 2007. Carbon storage in Larrea divaricata and L. cuneifolia (Zygophyllaceae) in drylands of Central.Western Argentina. Arid Land Res. Manag. 21: 273-285.

NEUMANN, R.P. \& G.E. MACHLIS. 1989. Land-use and threats to parks in the neotropics. Environ. Conserv. 16: 13-18.

NI, J. 2003. Plant functional types and climate along a precipitation gradient in temperate grasslands, northeast China and south-east Mongolia. J. Arid Environ. 53: 501-516.

PASSERA C.B., A.D. DALMASSO \& O. BORSETTO 1983. Metodo de Point Quadrat Modificado. En: Taller de arbustos forrajeros para zonas áridas y semiáridas. Subcomité Asesor del Árido Subtropical Argentino, IADIZA, Mendoza.

REYNOLDS, J.F., F.T. MAESTRE., E. HUBERSANNWALD, J. HERRICK \& P.R KEMP. 2005. Aspectos socioeconómicos y biofísicos de la desertificación. Ecosistemas 14: 3-21.

ROIG, F. \& E. MARTÍNEZ CARRETERO. 1998. La vegetación puneña en la provincia de Mendoza, Argentina. Phytocoenologia 28: 565-608.

ROIG, F., S. ROIG-JUÑENT \& V. CORVALÁN. 2009. Biogeography of the Monte Desert. J. Arid Environ. 73: $164-172$.

SANDERSON, E.W., M.A. JAIDEH, K.H. LEVY, K. REDFORD, A.W. WANNEBO \& G. WOLVER. 2002. The human footprint and the last of the wild. Bioscience 52: 891-904.

SCHUMAN, G.E., H.H. JANZEN \& J.E. HERRICK. 2002. Soil carbon dynamics and potential carbon sequestration by rangelands. Environ. Pollut. 116: 391-396.

SMITH, T.M., H.H. SHUGART \& F.I. WOODWARD (Eds.). 1997. Plant Functional Types: Their Relevance to Ecosystem Properties and Global Change. Cambridge University Press, New York. 


\section{Zivkovic et al. - Carbono acumulado en la biomasa vegetal de Mendoza}

UNEP-WCMC. 2008. Carbon and Biodiversity: a demonstration atlas. Kapos, V., C. Ravilious, A. Campbell, B. Dickson, H. Gibbs, M. Hansen, I. Lysenko, L. Miles, J. Price, J.P.W. Scharlemann J.P.W. \& K. Trumper (Eds.). UNEP-WCMC, Cambridge.

VERÓN, S.R., J.M. PARUELO \& M. OESTERHELD. 2006. Assessing desertification. J. Arid Environ. 66: 751-763.

WATSON, R.T., I.R. NOBLE, B. BOLIN, N.H. RAVINDRANATH, D.J. VERADO \& D.J. DOKKEN (Eds). 2000. Land Use, Land-use Change, and Forestry. Intergovernmental Panel for Climate Change, Cambridge University Press, Cambridge. WOOMER, P.L., L.L. TIESZEN, G. TAPPAN, A. TOURÉ \& M. SALL. 2004. Land use change and terrestrial carbon stocks in Senegal. J. Arid Environ. 59: 625-642.

Recibido el 16 de noviembre de 2012, aceptado el 29 de mayo de 2013. 
